\title{
BMJ Open Study protocol of the BLANKET trial: a cluster randomised controlled trial on the (cost-) effectiveness of a primary care intervention for fear of cancer recurrence in cancer survivors
}

\author{
Yvonne L Luigjes-Huizer (1) , ${ }^{1,2}$ Marije L van der Lee, ${ }^{1}$ Niek J de Wit, ${ }^{2}$ \\ Charles W Helsper ${ }^{2}$
}

To cite: Luigjes-Huizer YL, van der Lee ML, de Wit NJ, et al. Study protocol of the BLANKET trial: a cluster randomised controlled trial on the (cost-) effectiveness of a primary care intervention for fear of cancer recurrence in cancer survivors. BMJ Open 2019;9:e032616. doi:10.1136/ bmjopen-2019-032616

- Prepublication history for this paper is available online To view these files, please visit the journal online (http://dx.doi org/10.1136/bmjopen-2019032616)

Received 27 June 2019 Revised 22 October 2019 Accepted 28 0ctober 2019

D) Check for updates

(C) Author(s) (or their employer(s)) 2019. Re-use permitted under CC BY-NC. No commercial re-use. See rights and permissions. Published by BMJ.

${ }^{1}$ Scientific Research, Helen Dowling Institute, Bilthoven, The Netherlands

${ }^{2}$ Julius Center for Health Sciences and Primary Care, University Medical Center Utrecht, Utrecht University, Utrecht, The Netherlands

Correspondence to Yvonne L Luigjes-Huizer; yluigjes@hdi.nl

\section{ABSTRACT}

Introduction Many successfully treated patients with cancer suffer from fear of cancer recurrence (FCR), affecting their quality of life and their physical, emotional, cognitive and social functioning. Effective psychological interventions for FCR exist but are not widely available, as they are typically offered by specialised psycho-oncology professionals and institutes. Concurrently, the role of primary care in cancer and survivorship care is increasing. Therefore, there could be a role for general practitioners (GPs) and mental health workers (MHWs) working in primary care in supporting patients with FCR. In the current study, the effectiveness of a primary care delivered FCR intervention will be evaluated.

Methods and analysis A two-armed cluster randomised trial will be conducted. The primary outcome will be FCR severity; secondary outcomes will be FCR-related distress, healthcare uptake and healthcare costs. Primary care practices in the Netherlands will be invited to participate in the study. Participating practices will be stratified by size and socioeconomic status and randomised. In the control arm, practices will provide care as usual. In the intervention arm, practices will offer the cognitivebehavioural FCR intervention that is being studied, which consists of an intake with the GP and five sessions with the MHW. Patients who have finished successful curative treatment for cancer between 3 months and 10 years ago will be invited to participate in the study by invitation letter from their GPs. Participating patients will fill out questionnaires at baseline, after 3 months and after 12 months. Data on healthcare use will be collected from their electronic health records. Qualitative interviews are held at $\mathrm{T} 1$ with patients and practitioners in the intervention group.

Ethics and dissemination The Medical Research Ethics Committee (METC) Utrecht has reviewed the study in accordance with the Dutch Medical Research Involving Human Subjects Act (WMO) and other applicable Dutch and European regulations. Based on the requirements of the WMO, the METC Utrecht has issued an approval of the above-mentioned study. Any protocol amendments will be communicated to all relevant parties. Written consent is obtained from study participants. Results will be dispersed
Strengths and limitations of this study

- A robust, pragmatic trial design reflecting daily care will be implemented in general practices.

- Quantitative and qualitative data are combined to provide comprehensive results.

- The intervention and trial were designed in close cooperation with patients and healthcare workers.

- A cluster randomised design, randomising at practice level, was required, since practitioners who have been trained on the intervention are unlikely to be able to provide usual care in the same way as before training.

- Patients are actively invited to participate in the study, making them less representative of the patients who currently seek care for FCR.

through peer-reviewed publications and scientific presentations.

Trial registration number NL7573 in the Netherlands Trial Register on 25-02-2019.

\section{INTRODUCTION}

Advances in the medical field have caused the number of cancer survivors to rise steadily in the past decades. ${ }^{1}$ With an increasing number of survivors, there is also an increasing need for survivorship care. ${ }^{2}$ A systematic review showed that fatigue, depression and anxiety are commonly reported in the 10 years after primary cancer treatment. ${ }^{3}$ Fear of cancer recurrence (FCR) is a more prevalent concern than any physical issue. ${ }^{2}$ In a study about unmet needs after breast cancer, FCR was the most reported need in all age groups $(38.2 \%)$, despite a relatively good prognosis. ${ }^{4}$

FCR has been defined as 'fear, worry, or concern relating to the possibility that cancer will come back or progress'. ${ }^{5}$ A review by Simard et al. found that an average of $73 \%$ 
of cancer survivors experience FCR; $49 \%$ experience a moderate to high level of FCR; and $7 \%$ experience a high level of FCR. ${ }^{6}$ FCR is a multidimensional construct, as demonstrated by the subscales of the Fear of Cancer Recurrence Inventory (FCRI): triggers, severity, psychological distress, coping strategies, functioning impairments, insight and reassurance. ${ }^{7}$ FCR exists on a scale from normal to clinical. ${ }^{8}$ In a 2-day colloquium with a group of experts and patient advocates, five preliminary categories of potential characteristics of clinical FCR were identified using the Delphi method. These are preoccupation with cancer return or progression, unhelpful coping strategies, impairments in daily functioning, great level of distress and limited ability to make plans. ${ }^{5}$

Many studies have explored factors that correlate with FCR development, with mixed results. The evidence for correlations between FCR and age, gender and physical symptoms is strongest, whereby younger patients, female patients and patients with more symptoms experience more FCR. ${ }^{6}$ In contrast, social support, optimism, having detailed information and being conscientious correlate with having less FCR. ${ }^{6910}$ Notably, associations between FCR and psychological factors (eg, metacognitions) are generally stronger than associations between FCR and demographic factors. ${ }^{11}$ FCR can persist for many years after the end of cancer treatment. ${ }^{612}$ There are also triggers that can temporarily increase FCR, including medical appointments, having unexplainable symptoms and hearing about cancer in the media. ${ }^{13}$

The impact of FCR varies. Having some FCR can be protective, if it leads to treatment compliance and healthy lifestyle adaptations. However, severe FCR can significantly decrease quality of life. ${ }^{14}$ Maladaptive coping styles include overuse of primary care for common acute symptoms, which can inadvertently augment fears and cause unnecessary healthcare costs, ${ }^{15}$ but also avoidance of social and healthcare appointments, risking delayed diagnosis of cancer recurrence. On average, healthcare uptake is increased for people with high FCR. ${ }^{16}$

A Danish study found that patients discussed social or psychological aspects of cancer, including FCR, more with family and friends than with their GP because they thought it was not the GP's mandate to address these concerns. ${ }^{17}$ In a Dutch study, $75 \%$ of patients' physical problems after having received a cancer diagnosis were discussed with GPs, compared with only one-third of emotional and social problems. ${ }^{18}$ When the need for psychosocial care is recognised, this positively affects quality of life, appreciation of care and communication with care providers. ${ }^{19}$ Therefore, it seems of added value if GPs assess the presence of FCR and refer to additional care when needed. ${ }^{20}$

Treating FCR is different from treating other anxiety disorders because FCR is not irrational, since the threat is actual and significant. ${ }^{21}$ Currently, there are different treatment options for FCR, which can be applied in a stepped care approach. The first level involves psychoeducation, normalisation and self-management. Next, cognitive-behavioural therapy (CBT), therapies focusing on acceptance ${ }^{22}$ and pharmacological treatment ${ }^{23}$ can be applied. In recent years, several trials have shown the effectiveness of new FCR interventions, ${ }^{24} 25$ including mindfulness programmes,${ }^{26-28}$ psychoeducation, ${ }^{29}$ CBT interventions, ${ }^{30-32}$ an intervention based on metacognitive therapy ${ }^{33}$ and a gratitude intervention. ${ }^{34}$ The Survivors Worries of Recurrent Disease (SWORD) study found that blended treatment with a specialised psychologist and an online FCR programme reduced FCR significantly more than usual care. ${ }^{32}$

Specialised psychological care for cancer is typically provided in hospitals and specialised institutes. Unfortunately, travel distance, limited energy of patients who had cancer and waiting lists counteract accessibility. ${ }^{35}$ Also, most cancer survivors do not require intensive specialised psychotherapy, but rather accessible psychological care. Online treatment is easily accessible and allows patients to obtain care when they feel fit enough and for a manageable duration. However, evidence of the effectiveness of completely self-guided interventions among patients with cancer with psychological distress is lacking. Some level of therapist involvement can help encourage engagement and promote adherence. ${ }^{36}$

Concurrently, cancer care and survivorship care are increasingly being provided in primary care because of patient preference, increasing numbers of patients with cancer and rising healthcare costs. ${ }^{1}$ Primary care is comprehensive, longitudinal and integrated, provided by teams of different professionals, ${ }^{1}$ increasingly including mental health professionals. ${ }^{37}$ Primary care providers generally have a long-standing relation with the patient. ${ }^{38} 39$ Patients view primary care staff as trusted professionals ${ }^{40}$ and prefer coming to primary care for anxiety issues because of practical reasons and stigma. ${ }^{41}$ General practitioners (GPs) want to provide psychosocial support to patients with cancer and feel they are well positioned, ${ }^{42}{ }^{43}$ but they face capacity challenges ${ }^{445}$ and report a need for training on cancer survivorship, ${ }^{46}{ }^{47}$ in particular, on treating psychological problems. ${ }^{44}$ Involving and training auxiliary staff, such as primary care mental health workers (MHWs), in survivorship care may help to overcome both capacity challenges and the need for improved expertise in primary care. ${ }^{47}$

\section{Aim}

The BLANKET (blended care for fear of cancer recurrence, Dutch acronym) study was designed to assess the effectiveness of a primary care delivered, blended care intervention for FCR, in reducing patients' severity of FCR, compared with usual care. Since this is a pragmatic trial, we include all patients who want care for FCR at their GP practice.

We hypothesise that

1. The FCR intervention will reduce FCR severity.

2. The FCR intervention will reduce FCR-related distress.

3. Healthcare consumption of patients who have received the FCR intervention will be reduced. 
4. The FCR intervention will be considered desirable and of added value by patients and practitioners.

The primary outcome is FCR severity. Secondary outcomes are FCR-related distress, FCR-related healthcare use, FCR-related health costs, and satisfaction of patients and practitioners with support provided by trained MHWs and GPs.

\section{METHODS}

\section{Study design}

The BLANKET study is a two-armed cluster randomised clinical trial in which the general practice is the unit of randomisation.

\section{Study procedure}

Participating practices will identify all of their patients who have successfully completed curative cancer treatment between 3 months and 10 years ago, and will send them an invitation letter by mail. Patients are asked to participate if they desire support for FCR. After providing informed consent, patients are asked to fill out an online baseline questionnaire. Patients also fill out questionnaires 3 and 12 months after baseline. At the end of the first questionnaire, they are urged to make an appointment with their GP about support for FCR. During this consultation, the GPs in the intervention group refer the patients to the MHW for the intervention, while GPs in the control group provide usual care.

\section{Eligibility}

Clusters of collaborating GPs and MHWs in the Netherlands who are willing to undergo training and to implement it will be recruited. In the Dutch setting, almost all general practices employ MHWs (in Dutch: POH-GGZ). ${ }^{48}$ Both a GP and an MHW need to agree to participate for the practice to be eligible to join the study.

Patients are eligible if they 1) are registered at a general practice that is participating in the study 2) are 18 years or older 3 ) have finished successful curative cancer treatment between 3 months and 10 years ago 4) desire support for FCR, and 5) have sufficient Dutch reading and writing skills to receive the intervention and to complete the questionnaires. If patients have a cancer recurrence during the study, no more data will be collected. GPs select patients who can be invited for the study. GPs exclude vulnerable patients (eg, severe psychiatric morbidity) who would be confused by the letter or unable to participate in the study.

Since this is a pragmatic real-world trial, we include all patients who want care for FCR at their GP practice. We chose not to screen for level of FCR as an inclusion criterion because this would not reflect daily practice. This intervention could also be relevant for patients with nonclinical levels of FCR who are nonetheless limited by FCR in daily life. We will train the MHW to refer patients who require specialised psychological care.

\section{Recruitment}

The aim is to include 244 patients during 1.5 years. Patients are recruited using an invitation letter sent by patients' own GPs. All of the patients of participating practices who are 18 years or older and have finished curative cancer treatment between 3 months and 10 years ago will receive the letter. To spread the workload for the practitioners, invitation will be done in rounds, starting with patients who most recently finished cancer treatment.

\section{Randomisation}

Randomisation is done at practice level. GPs and MHWs will know in which group they have been placed. Patients will not. Clusters are formed, in which all GPs and MHWs working in the same building are grouped together to decrease the risk of contamination. Minimisation is applied for size of the practice and the socioeconomic status (SES) of the neighbourhood they are located in, to ensure balance between study arms (patients and professionals). For practice size, there are three categories: small (one to three GPs), middle-sized (four to six GPs) or large (seven GPs or more). For SES, the list of disadvantaged areas by postal code made by the Dutch government for general practices is used. Practices will be assigned to the intervention or the control group using the number generator at Research Randomizer ( randomizer.org). An external data manager will generate the numbers. Practices are randomised in two blocks. The inclusion rate from the first block will help to confirm how many more practices are needed for the second block.

\section{Intervention}

GPs and MHWs in the intervention group will provide an intervention specifically designed for FCR, with online modules that focus on normalisation, psychoeducation and self-management. ${ }^{49}$ The modules were developed at the Helen Dowling Institute based on CBT, clinical experience and input from patients, and are currently being used by specialised psychologists for blended treatment. The intervention consists of two CBT modules, which include psychoeducation on FCR, and five optional modules on rumination, avoidance, relaxing, reassuring and undertaking activities. The FCRI is used to determine which optional modules are most important for each patient. Patients can also choose additional modules.

GPs in the intervention group will undergo a 1-hour online training. MHWs in the intervention group will undergo two 2-hour training sessions by an experienced clinical psychologist, including role plays with an actor playing a patient. The trainings will be about FCR and how to provide the intervention. In between sessions, the MHWs will practice using the online modules, both as a patient and as a practitioner. In providing the intervention, the GP's role is to assess the need for care during an intake and to refer to the MHW. The MHW's role is to assign and discuss the modules with the patients during five contact moments. MHWs will openly listen to the patients' experiences, normalise fears, apply CBT and discuss what was gained from the modules. Any related questions and issues that come up will also be discussed. 
GPs and MHWs in the control group will provide usual care.

\section{Usual care}

Patients in the control group receive usual care. In the literature, little is known about the usual care that GPs provide for FCR. Therefore, usual care will be mapped in this study, in relation to the severity of FCR.

\section{Outcomes}

Participants will provide data using online self-report questionnaires hosted by ResearchOnline.com. Participants will receive an invitational email with a link to complete the questionnaires online. These links will be sent at baseline (T0), after 3 months, once the intervention in the intervention group is completed (T1) and 1 year after the baseline (T2). Participants who do not respond will receive reminders. If participants prefer to fill out the questionnaires on paper, this will be arranged. If patients do not fill out the questionnaires, they will be sent reminders.

\section{Primary outcome}

The primary outcome is the severity of FCR after 3 months, comparing the FCR intervention with usual care. To measure this, the severity scale (SF) of the Dutch version of the Fear of Cancer Recurrence Inventory (FCRI-NL) will be used.

\section{Secondary outcomes}

The secondary outcomes are the development from baseline to T1 to T2 of the severity of FCR, FCR-related distress, FCR-related healthcare use and FCR-related health costs, and the desirability and added value of the intervention.

\section{Covariates}

If the intervention is found to be effective, relations between the outcomes and the following variables will be explored to identify groups of patients for whom the intervention might be more or less effective.

Covariates at the patient level include age, gender, level of education, coping style, severity of anxiety and depression, somatic complaints, severity of FCR at the start of the study, FCR-related distress at the start of the study, psychiatric history, previous healthcare use, additional care used by patients (eg, alternative care), time since the cancer diagnosis, time since the end of the curative cancer treatment and cancer type.

Covariates at the practice level include the general practice size and the SES status of the practice.

Covariates at the MHW level include the number of years of work experience and the educational background of the MHW.

\section{Data collection}

Patients will fill out the FCRI-NL. It contains 43 items measuring seven subscales. The severity, distress and coping subscales will be used to measure FCR severity, distress and coping. The FCRI was translated into Dutch and validated by van Helmondt et $a l^{50}{ }^{50}$ While for the FCRI it is recommended to use the total score of all subscales to obtain a score for $\mathrm{FCR}^{7}$ this multidimensional structure was not replicated in the validation of the FCRI-NL. Instead, the individual subscales provide important information and are recommended to be used separately. ${ }^{50}$

The Four-Dimensional Symptom Questionnaire (4DSQ) will be used to provide data on general distress, depression, anxiety and somatic complaints. The $4 \mathrm{DSQ}$ is a Dutch 50-item questionnaire that measures four dimensions: distress, depression, anxiety and somatic complaints. The list is already used in some GP practices and is therefore practically applicable.

Patients will also be surveyed about their educational level, current daily activity (eg, work), reasons for participating in the study, additional care used that is not in the electronic health records (EHRs), including alternative care, and other factors that they think might have influenced their FCR.

In order to collect data on patients' cancer type, treatment and healthcare use, data will be obtained from patients' EHR. Data will be collected on the number of GP visits related to cancer, FCR and neither, the number of sessions with the MHW, and the number of referrals for physical care and for psychological care. GP visits will only be considered FCR related if FCR is specifically mentioned. Some patients may not mention FCR but have increased healthcare uptake due to hypervigilance. If that is the case, we expect the number of cancer-related visits to decrease if FCR decreases. At baseline, data on healthcare use per year since the end of curative cancer treatment will also be obtained to exploratively compare usual care in our control group with usual care in the years prior to the study. FCR-related health costs will be calculated based on the healthcare use.

The desirability and added value of the intervention will be evaluated using custom-made, non-validated questionnaires and semistructured interviews with a selection of patients and practitioners at $\mathrm{T} 1$. The interviews will explore which aspects of the support are effective, unnecessary, practical or pleasant and why. They will also explore whether the GP and MHW are considered to be the right practitioners to provide this type of care and what changes with regard to FCR are most desirable and sought after. Varied groups will be purposively sampled: for patients, in terms of age, time since diagnosis, severity of FCR at T0, and severity of FCR at T1; and for practitioners, in terms of professional background and years of work experience.

Additional information about data collection, data management, monitoring and dissemination of results can be found in the trial master file.

\section{Sample size calculation}

When determining the required group size for finding a relevant difference between the groups, we used a difference in means of 3 and an SD of 7 on the FCRI-SF. The 
difference in means was based on expert opinion. The SD was based on the FCRI-NL validation study by van Helmondt et al, ${ }^{50}$ which found an SD of 7 on the SF. ${ }^{50}$ Using an alpha of 0.05 and a beta of 0.8 , we calculated a required sample size of 86 participants in both groups for sufficient power. Because multiple patients are treated by the same MHW, there might be a cluster effect. Based on an average of 15 inclusions per MHW and an intraclass correlation coefficient of 0.01 , an inflation factor of 1.14 has been applied. This leads to a group size of 98 patients per arm. Because the clusters (number of patients per MHW) will probably not all have the same size, an inflation factor of $10 \%$ is applied, leading to a group size of 108 . We also assume a dropout of $12 \%$ of patients. That is why we aim to include 122 patients in each group.

\section{Statistical analysis}

The primary outcome will be expressed as difference in the mean (with $95 \% \mathrm{CI}$ and $\mathrm{p}$ value) of the $\mathrm{SF}$ of the FCRI-NL scale between the intervention and control groups at $\mathrm{T} 1$.

This will be analysed with a linear mixed model. A random intercept will be included to correct for inclusion per MHW. We will include residual covariances in order to correct for repeated measurement in each patient.

The analyses will be conducted in two steps. First, an analysis will be performed with time, treatment and a time by treatment interaction. Second, a correction for baseline measurement of the outcome will be added to the first model.

The validity of the models will be assessed with residual analyses. $^{51}$

A similar approach will be used to analyse secondary outcomes and covariates. Where applicable, a generalised linear model will be used to analyse dichotomous and count outcomes (for binomial and Poisson distributions, respectively).

Healthcare use is analysed using multilevel analyses. The number of visits to the GP between $\mathrm{T} 1$ and $\mathrm{T} 2$ is compared between the intervention group and the control group. Shifts in type of visits-physical versus psychological-will also be explored. The healthcare uptake in the control group between $\mathrm{T} 1$ and $\mathrm{T} 2$ will also be compared with the period before the baseline measurement to assess whether healthcare uptake has changed since participating in the study.

The costs of healthcare are compared between the control group and the intervention group for the period between $\mathrm{T} 0$ and $\mathrm{T} 1, \mathrm{~T} 1$ and $\mathrm{T} 2$, and $\mathrm{T} 0$ and $\mathrm{T} 2$, whereby the period between $\mathrm{T} 0$ and $\mathrm{T} 2$ is most important since it combines the costs of the intervention and the potential change in costs in the 9 months after the intervention. Healthcare costs are calculated based on healthcare use, according to the method of the Guidelines for Carrying Out Economic Evaluations in Healthcare. ${ }^{52}$

For the outcomes for which the intervention is found to be effective, the effect of the covariates on the outcomes will be explored. First, intention-to-treat analyses will be done. Then, per-protocol analyses will be carried out to estimate the effectiveness of the intervention if executed per protocol. During the analyses, the assessor will be blinded about the groups.

The validity of the study results may be challenged by missing values, either at baseline or missing outcomes at follow-up. Multiple imputation will be used to address missing values at baseline for relevant variables. For missing outcomes, correction for relevant prognostic factors will be considered to ensure the validity of the results. ${ }^{53}$

The desirability and feasibility of the intervention according to patients and practitioners will be measured qualitatively. Semistructured interviews will be held. These will be transcribed and then coded by two independent researchers. Differences in coding will be discussed until consensus is reached. Important themes will be identified using the data as the starting point.

\section{Patient and public involvement}

When developing the intervention, patients provided input on the desired content and appearance, for example, preference for short texts. Once implemented, the intervention was further adapted based on patient feedback.

When developing the study, patients provided input on the general idea. They also provided feedback on the recruitment process and, in particular, on the invitation letter to patients. Based on their input, the study and the letter were adapted.

\section{DISCUSSION}

With an increased number of cancer survivors, there is an increased need for survivorship care. Providing survivorship care in primary care may improve access and reduce the pressure on specialised institutions. In this study, the effectiveness of a primary care FCR intervention will be compared with usual care. An evaluation of healthcare consumption and costs will assess whether this can also decrease healthcare uptake and costs. To our knowledge, this is the first trial assessing the effectiveness of a primary care FCR intervention. In addition, it is one of few pragmatic trials on FCR interventions.

\section{Heterogeneity of usual care}

To assess whether this intervention is more effective than what is currently being offered, we choose to compare with usual care. No clear guidelines are available for GPs for FCR, so usual care may be quite diverse. Therefore, we will register usual care during the study.

\section{Recruitment}

Because prior research shows that patients often do not mention FCR to their GP, we choose to actively invite patients to participate in the study. The disadvantage of this choice is that we are activating our participants, making them less representative of the patients who 
currently seek care for FCR. However, we want to know whether this intervention can help patients with FCR, if they choose to seek care.

\section{Usual care}

We recognise that the usual care measured in this study might not fully reflect actual usual care, since we have activated the patient population and made the general practices more aware of this issue. To assess the effect of this activation, we compare the healthcare use in the control group with retrospective healthcare use. Also, practices who agree to participate in the study might have increased interest and expertise in FCR. To assess this, we ask them about any education on FCR or related topics they have received.

\section{Randomisation level}

We choose to randomise practices and not patients to prevent contamination. Practitioners who have been trained will have increased knowledge and awareness and will no longer provide usual care the way they did before training. Also, patients at the same practice might discuss the intervention they receive and notice the differences. Patients are unaware of the randomisation to prevent patients in the control group from being disappointed and less motivated.

\section{Trial status}

Invitation of primary care practices started in October 2018. The first patient was included on 15 April 2019. Final results are expected in 2020.

Contributors All authors participated in the design of the study. YLL wrote the draft of the manuscript. MLvdL, CWH and NJdW improved the manuscript. All authors read and approved the final manuscript.

Funding This work was supported by the Dutch Cancer Society (KWF) grant number 10936. KWF is not involved in study design, collection, management, analysis and interpretation of data, writing of the report and decision to submit the report for publication, nor does it have authority over the publications. Sponsor: Helen Dowling Institute, Professor Bronkhorstlaan 20, 3723 MB Bilthoven.

Competing interests None declared.

Patient consent for publication Not required.

Provenance and peer review Not commissioned; externally peer reviewed.

Open access This is an open access article distributed in accordance with the Creative Commons Attribution Non Commercial (CC BY-NC 4.0) license, which permits others to distribute, remix, adapt, build upon this work non-commercially, and license their derivative works on different terms, provided the original work is properly cited, appropriate credit is given, any changes made indicated, and the use is non-commercial. See: http://creativecommons.org/licenses/by-nc/4.0/.

ORCID iD

Yvonne L Luigjes-Huizer http://orcid.org/0000-0003-1611-3627

\section{REFERENCES}

1 Rubin G, Berendsen A, Crawford SM, et al. The expanding role of primary care in cancer control. Lancet Oncol 2015;16:1231-72.

2 Ness S, Kokal J, Fee-Schroeder K, et al. Concerns across the survivorship trajectory: results from a survey of cancer survivors. Oncol Nurs Forum 2013;40:35-42.

3 Harrington CB, Hansen JA, Moskowitz M, et al. It's not over when it's over: long-term symptoms in cancer survivors--a systematic review. Int J Psychiatry Med 2010;40:163-81.
4 Brennan ME, Butow P, Spillane AJ, et al. Patient-reported quality of life, unmet needs and care coordination outcomes: moving toward targeted breast cancer survivorship care planning. Asia Pac J Clin Oncol 2016;12:e323-31.

5 Lebel S, Ozakinci G, Humphris G, et al. From normal response to clinical problem: definition and clinical features of fear of cancer recurrence. Support Care Cancer 2016;24:3265-8.

6 Simard S, Thewes B, Humphris G, et al. Fear of cancer recurrence in adult cancer survivors: a systematic review of quantitative studies. $J$ Cancer Surviv 2013;7:300-22.

7 Simard S, Savard J. Fear of cancer recurrence inventory: development and initial validation of a multidimensional measure of fear of cancer recurrence. Support Care Cancer 2009;17:241-51.

8 Simard S, Savard J. Screening and comorbidity of clinical levels of fear of cancer recurrence. J Cancer Surviv 2015;9:481-91.

9 Hedman C, Strang P, Djärv T, et al. Anxiety and fear of recurrence despite a good prognosis: an interview study with differentiated thyroid cancer patients. Thyroid 2017;27:1417-23.

10 Liao KY-H, Yeung NCY, Wong CCY, et al. Fear of cancer recurrence and physical well-being among Chinese cancer survivors: the role of conscientiousness, positive reappraisal and hopelessness. Support Care Cancer 2017;25:1141-9.

11 Smith A, Sharpe L, Thewes B, et al. Medical, demographic and psychological correlates of fear of cancer recurrence (FCR) morbidity in breast, colorectal and melanoma cancer survivors with probable clinically significant FCR seeking psychological treatment through the ConquerFear study. Support Care Cancer 2018;26:4207-16.

12 Simard S, Savard J, Ivers H. Fear of cancer recurrence: specific profiles and nature of intrusive thoughts. $J$ Cancer Surviv 2010;4:361-71.

13 Gill KM, Mishel M, Belyea M, et al. Triggers of uncertainty about recurrence and long-term treatment side effects in older African American and Caucasian breast cancer survivors. Oncol Nurs Forum 2004;31:633-9.

14 Simonelli LE, Siegel SD, Duffy NM. Fear of cancer recurrence: a theoretical review and its relevance for clinical presentation and management. Psychooncology 2017;26:1444-54.

15 Butow P, Sharpe L, Thewes B, et al. Fear of cancer recurrence: a practical guide for clinicians. Oncology 2018;32:32-8.

16 Champagne A, Ivers H, Savard J. Utilization of health care services in cancer patients with elevated fear of cancer recurrence. Psychooncology 2018;27:1958-64.

17 Mikkelsen T, Sondergaard J, Sokolowski I, et al. Cancer survivors' rehabilitation needs in a primary health care context. Fam Pract 2009;26:221-30.

18 NIVEL. Afstemming van zorg tussen huisarts en specialist bij kanker kan beter [Internet], 2013. Available: https://www.nivel.nl/nl/nieuws/ afstemming-van-zorg-tussen-huisarts-en-specialist-bij-kanker-kanbeter [Accessed 22 May 2018].

19 NHG. NHG-standpunt Oncologische zorg in de huisartsenpraktijk, 2014.

20 Fann JR, Ell K, Sharpe M. Integrating psychosocial care into cancer services. J Clin Oncol 2012;30:1178-86.

21 Curran L, Sharpe L, Butow P. Anxiety in the context of cancer: a systematic review and development of an integrated model. Clin Psychol Rev 2017;56:40-54.

22 Völker $\mathrm{C}$, van der Lee M, Pet A. De angst voor terugkeer van kanker. GZ - Psychologie 2011;3:30-8.

23 Cupit-Link M, Syrjala KL, Hashmi SK. Damocles' syndrome revisited: update on the fear of cancer recurrence in the complex world of today's treatments and survivorship. Hematol Oncol Stem Cell Ther 2018;11:129-34.

24 Chen D, Sun W, Liu N, et al. Fear of cancer recurrence: a systematic review of randomized, controlled trials. Oncol Nurs Forum 2018;45:703-12.

25 Sharpe L, Thewes B, Butow P. Current directions in research and treatment of fear of cancer recurrence. Curr Opin Support Palliat Care 2017;11:191-6.

26 Crane-Okada R, Kiger H, Sugerman F, et al. Mindful movement program for older breast cancer survivors: a pilot study. Cancer Nurs 2012;35:E1-13.

27 Lengacher CA, Shelton MM, Reich RR, et al. Mindfulness based stress reduction (MBSR $(B C)$ ) in breast cancer: evaluating fear of recurrence (FOR) as a mediator of psychological and physical symptoms in a randomized control trial (RCT). J Behav Med 2014;37:185-95.

28 Lengacher CA, Reich RR, Paterson CL, et al. Examination of broad symptom improvement resulting from mindfulness-based stress reduction in breast cancer survivors: a randomized controlled trial. $J$ Clin Oncol 2016;34:2827-34. 
29 Dieng M, Butow PN, Costa DSJ, et al. Psychoeducational intervention to reduce fear of cancer recurrence in people at high risk of developing another primary melanoma: results of a randomized controlled trial. J Clin Oncol 2016;34:4405-14.

30 Dodds SE, Pace TWW, Bell ML, et al. Feasibility of CognitivelyBased compassion training (CBCT) for breast cancer survivors: a randomized, wait list controlled pilot study. Support Care Cancer 2015;23:3599-608.

31 Lichtenthal WG, Corner GW, Slivjak ET, et al. A pilot randomized controlled trial of cognitive bias modification to reduce fear of breast cancer recurrence. Cancer 2017;123:1424-33.

32 van de Wal M, Thewes B, Gielissen M, et al. Efficacy of blended cognitive behavior therapy for high fear of recurrence in breast, prostate, and colorectal cancer survivors: the sword study, a randomized controlled trial. J Clin Oncol 2017;35:2173-83.

33 Butow PN, Turner J, Gilchrist J, et al. Randomized trial of ConquerFear: a novel, theoretically based psychosocial intervention for fear of cancer recurrence. $J$ Clin Oncol 2017;35:4066-77.

34 Otto AK, Szczesny EC, Soriano EC, et al. Effects of a randomized gratitude intervention on death-related fear of recurrence in breast cancer survivors. Health Psychol 2016;35:1320-8.

35 Zernicke KA, Campbell TS, Speca M, et al. A randomized wait-list controlled trial of feasibility and efficacy of an online mindfulnessbased cancer recovery program: the eTherapy for cancer applying mindfulness trial. Psychosom Med 2014;76:257-67.

36 Ugalde A, Haynes K, Boltong A, et al. Self-guided interventions for managing psychological distress in people with cancer - A systematic review. Patient Educ Couns 2017;100:846-57.

37 Harkness EF, Bower PJ, Elaine FH. On-site mental health workers delivering psychological therapy and psychosocial interventions to patients in primary care: effects on the professional practice of primary care providers. Cochrane Database Syst Rev 2009.

38 Del Giudice ME, Grunfeld E, Harvey BJ, et al. Primary care physicians' views of routine follow-up care of cancer survivors. J Clin Oncol 2009;27:3338-45.

39 Johansen M-L, Holtedahl KA, Rudebeck CE. How does the thought of cancer arise in a general practice consultation? Interviews with GPs. Scand J Prim Health Care 2012;30:135-40.

40 Mitchell AJ, Vahabzadeh A, Magruder K. Screening for distress and depression in cancer settings: 10 lessons from 40 years of primarycare research. Psychooncology 2011;20:572-84.
41 Curran GM, Sullivan G, Mendel P, et al. Implementation of the calm intervention for anxiety disorders: a qualitative study. Implement Sci 2012;7:1-11.

42 Lawrence RA, McLoone JK, Wakefield CE, et al. Primary care physicians' perspectives of their role in cancer care: a systematic review. J Gen Intern Med 2016;31:1222-36.

43 Berrett-Abebe J, Cadet T, Vitello J, et al. Developing content for an interprofessional training on fear of cancer recurrence (FCR): key informant interviews of healthcare professionals, researchers and cancer survivors. J Psychosoc Oncol 2018;36:259-73.

44 Berrett-Abebe J, Cadet T, Nekhlyudov L, et al. Impact of an interprofessional primary care training on fear of cancer recurrence on clinicians' knowledge, self-efficacy, anticipated practice behaviors, and attitudes toward survivorship care. J Cancer Educ 2019;34:505-11.

45 Fidjeland HL, Brekke M, Vistad I. General practitioners' attitudes toward follow-up after cancer treatment: a cross-sectional questionnaire study. Scand J Prim Health Care 2015;33:223-32.

46 Nekhlyudov L, O'malley DM, Hudson SV. Integrating primary care providers in the care of cancer survivors: gaps in evidence and future opportunities. Lancet Oncol 2017;18:e30-8.

47 Adam R, Watson E. The role of primary care in supporting patients living with and beyond cancer, 2018: 261-7. www.supportiveandpal liativecare.com

48 Landelijke verening POH-GGZ. Functie-en competentieprofiel $\mathrm{POH}$ GGZ, 2014: 17.

49 van Helmondt SJ, van der Lee ML, de Vries J. Study protocol of the CAREST-trial: a randomised controlled trial on the (cost-) effectiveness of a CBT-based online self-help training for fear of cancer recurrence in women with curatively treated breast cancer. BMC Cancer 2016;16:1-11.

50 van Helmondt SJ, van der Lee ML, de Vries J. Translation and validation of the Dutch version of the fear of cancer recurrence inventory (FCRI-NL). J Psychosom Res 2017;102:21-8.

51 Fitzmaurice GM, Laird NM, Ware JH. Applied longitudinal analysis. 2nd edn. Hoboken: John Wiley \& sons, 2004.

52 Assessment MT. Verdiepingsmodule Kostenevaluatie. Available: https://www.zorginstituutnederland.nl/publicaties/publicatie/2016/02/ 29/richtlijn-voor-het-uitvoeren-van-economische-evaluaties-in-degezondheidszorg

53 Groenwold RHH, Moons KGM, Vandenbroucke JP. Randomized trials with missing outcome data: how to analyze and what to report. CMAJ 2014;186:1153-7. 